\title{
Bringing Liraglutide to Market: A CER Case Study
}

\author{
Gary Oderda, PharmD, MPH, and S. Monet Sifford-Wilson, MD
}

\begin{abstract}
BACKGROUND: Faced with competition from other drugs and therapies, drug manufacturers may be able to use comparative effectiveness research (CER) to help reduce barriers to a new drug's adoption and integration into formularies. But few examples exist to show how CER can be used effectively and whether the data can make a difference.

OBJECTIVES: To examine how CER can help strengthen a new drug's entry into the market and integration into formularies, and how ongoing CER might be valuable as a drug is implemented in the real world.

SUMMARY: A roundtable of 9 representatives from health plans, including formulary decision makers, evaluated how CER in phase 3 development of a new drug can add to the drug's strength of evidence, helping decision makers understand how and where to integrate that drug into a formulary. The round table participants viewed, as a case study, the development of liraglutide, a glucagon-like peptide-1 (GLP-1) receptor agonist for adults with type 2 diabetes that was approved by the FDA in January 2010. With this drug, CER was incorporated into an extensive type 2 diabetes clinical development program, comparing how the drug worked in comparison with other established therapies. Although there are many antidiabetic drugs available for use, patients with type 2 diabetes often need additional agents. The FDA approved liraglutide with the conclusion that benefits of the drug outweighed potential risks but noted the association with pancreatitis in humans and animal data that showed rare medullary thyroid cancer associated with liraglutide. Roundtable participants agreed that while prelaunch CER can be valuable, ongoing real-world research is also important for confirming expected results, identifying additional uses and indications and managing risks. The participants also suggested opportunities for additional CER studies and made recommendations for manufacturers.

CONCLUSIONS: Roundtable thought leaders agreed that well-planned trial designs incorporating CER result in high-quality evidence that may provide sufficient data to support adoption of a new therapy onto the formulary. When more real-world data become available and confirm the phase 3 clinical trial results, decision makers may be able to use the results to change the drug's position and either lessen or extend its use.
\end{abstract}

J Manag Care Pharm. 2012;18(5):S12-S18

Copyright @2012, Academy of Managed Care Pharmacy. All rights reserved.

\section{What is already known about this subject}

- For FDA registration in the United States, it is common to demonstrate efficacy of a new product by comparing the new product with a placebo rather than using head-to-head comparisons with clinically relevant alternatives. Without a requirement for clinical trials using active comparators, many manufacturers opt to avoid the extra expense and risk of unfavorable results in these types of studies

- Comparative effectiveness research (CER) may help reduce barriers to a new drug's adoption and integration into formularies. The safety and efficacy of liraglutide were investigated in an extensive clinical development program in adults with type 2 diabetes and showed that the benefits from the drug outweighed the risks.

\section{What this article adds}

- It was a consensus of the 9 roundtable participants that wellplanned trial designs that result in a broad range of relevant data, such as that presented for liraglutide, can provide a good basis for the decision maker.

- Even if a drug comes to market with a solid set of CER data, roundtable participants felt that additional post-launch comparative data, such as studies showing how the drug might benefit subpopulations or studies pursuing alternative combination therapies or other comparators, might even strengthen the case for the drug.

- The roundtable participants recommended that manufacturers should translate CER and clinical data into tools that will enable decision makers to create effective benefit designs and make good formulary decisions; that manufacturers be proactive in communicating with all stakeholders to keep them informed of ongoing data (especially with safety issues); and that manufacturers should pursue opportunities to validate data from randomized controlled trials in the real world.

$\mathrm{T}$ reatment of type 2 diabetes mellitus includes several classes of drugs with multiple agents in each class that are generally safe and effective, yet the proportion of patients at goal is suboptimal. There still remains an unmet need for drugs to treat type 2 diabetes with better overall riskbenefit profiles, especially in light of the weight gain and hypoglycemia frequently seen with some commonly used agents.

As Saydah et al. (2004) point out, only 37\% of diagnosed diabetic participants were at the American Diabetes Association goal of hemoglobin Alc level of less than $7.0 \%$ in the 1999-2000 NHANES cohort, but this improved to $57.1 \%$ in the 2003-2004 NHANES sample., ${ }^{1,2}$ Successful introduction of a new drug into this market requires data that the new drug can demonstrate an improvement upon existing therapies for at least some portion of the population.

Comparative effectiveness research (CER) may help reduce barriers to a new drug's adoption and integration into formularies. But according to Hochman and McCormick (2010), who evaluated all human studies published between June 2008 and September 2009 in 6 key general and internal medicine journals (New England Journal of Medicine, Lancet, JAMA, Annals of Internal Medicine, BMJ, and Archives of Internal Medicine), most of the published research does not fulfill the criteria of CER, and much of the study design is driven by U.S. Food and Drug Administration (FDA) requirements rather than the need for evidence allowing the selection of the most effective therapy. ${ }^{3}$

At the October 2010 Academy of Managed Care Pharmacy (AMCP) meeting, a payer executive roundtable was convened, comprising 9 representatives from a broad spectrum of health plan types, including Medicaid, those providing Medicare 
Part D drug formularies, and drug formularies as defined by contracts with health plans or employers. This roundtable of thought leaders was invited to examine the case study of liraglutide [rDNA origin] injection (Victoza), a glucagon-like peptide-1 (GLP-1) receptor agonist approved by the FDA on January 25, 2010, with the indication as an adjunct to diet and exercise to improve glycemic control in adults with type 2 diabetes. ${ }^{4}$ The label lists the following as "important limitations of use:"

- "Not recommended as first-line therapy for patients inadequately controlled on diet and exercise.

- Has not been studied sufficiently in patients with a history of pancreatitis. Use caution.

- Not for treatment of type 1 diabetes mellitus or diabetic ketoacidosis.

- Has not been studied in combination with prandial insulin." ${ }^{4}$

Subsequent to the conduct of the executive roundtable, the warnings and precautions section of the label was updated in May 2011 regarding renal impairment, that "has been reported postmarketing, usually in association with nausea, vomiting, diarrhea, or dehydration which may sometimes require hemodialysis. Use caution when initiating or escalating doses of Victoza in patients with renal impairment."

After viewing the evidence from 6 clinical trials, roundtable participants offered their perspectives on the strength of the CER data reported in this case and how it would affect their decisions about the drug. In the phase 3 clinical trial program, the randomized portions of all phase 3 a trials (LEAD 1-5) were double-blind (except the insulin glargine arm of LEAD-5), placebo-controlled (except LEAD-3), active-comparator (except LEAD-4), parallel-group studies. In addition, participants offered ideas for how continuing real-world CER might be valuable for future decisions about a drug post-launch.

\section{Incorporating CER into Clinical Trials: Beyond the Norm}

Launching a new drug into a therapy area that already contains existing effective treatments can be challenging. Without comparative effectiveness data that demonstrates how the new drug works versus those existing treatments, formulary decision makers may not see any compelling reasons to help them decide where or even whether to integrate the new drug into their formularies. The drug development process involves 4 general phases of human trials. Phase 1 trials administer the drug to a small number of healthy volunteers to study activity and safety. In phase 2 the focus is on effectiveness, and a few hundred volunteers with the disease of interest are treated. Phase 3 trials generally involve several thousand patients with the disease of interest, are the primary trials used for registration, and they evaluate both efficacy and safety. Phase 4 trials are done after the FDA has approved the drug for marketing and look at long-term efficacy and safety in real-world patients. Incorporating CER into the phase 3 development of a new drug, especially an innovative drug, may provide the compelling data necessary to persuade decision makers.

For FDA registration in the United States, it is common to demonstrate efficacy of a new product by comparing the new product with a placebo using a pivotal phase 3 trial.,5 Headto-head comparisons with clinically relevant alternatives are not done in most cases. Without a requirement for CER-based studies, manufacturers may opt to avoid the extra expense and risk of these types of studies. However, some manufacturers are beginning to see value in such studies, especially if the data shows formulary decision makers that the new drug presents a clear advantage in at least some populations.

Safety and Efficacy of Liraglutide. The safety and efficacy of liraglutide were investigated in one of the most extensive clinical development programs ever conducted in type 2 diabetes.-13 The phase 3 clinical trials for the drug included 4,445 patients and comprised 5 randomized, placebo-controlled, double-blind trials plus 1 open-label trial (liraglutide vs. exenatide). ${ }^{14}$ There was 1 monotherapy study of liraglutide versus the sulfonylurea glimepiride of 52-week duration and 5 combination-therapy studies of 26-week duration. ${ }^{7-12}$ All 6 trials were randomized, multicenter, parallel-group design, and all trials were doubleblinded except the metformin plus glimepiride add-on trial, which included open-label insulin glargine and the metformin and/or glimepiride add-on open-label trial versus exenatide. ${ }^{11}$

In addition, the monotherapy trial and the combination therapy trials with metformin or glimepiride add-ons were double-dummy design. The program compared once-daily liraglutide injections with 3 widely used diabetes therapiesglimepiride, rosiglitazone, and insulin glargine-and 1 study also directly compared liraglutide with exenatide. ${ }^{7-12}$ In addition, one 26-week, randomized, parallel-group, open-label head-to-head trial with sitagliptin was performed. ${ }^{13}$

The studies were designed to investigate the efficacy and safety of liraglutide at each step in the treatment continuum, from monotherapy to combination with 2 oral antidiabetic drugs in adults with type 2 diabetes. All but 1 of the studies had an active comparator. A substantial effect on Alc reduction, combined with significant weight reduction and low risk for hypoglycemia, was consistently shown.

Zinman et al. (2012) conducted a meta-analysis of the Liraglutide Effect and Action in Diabetes (LEAD) trials using a composite endpoint (Alc $<7 \%$ with no weight gain and no hypoglycemia) to compare liraglutide to other agents from standard classes of antidiabetic therapy. ${ }^{15}$ The composite endpoint at 26 weeks was attained in $40 \%$ of the liraglutide 1.8 milligram (mg) group, 32\% of the liraglutide $1.2 \mathrm{mg}$ group and $6 \%-25 \%$ of comparators (6\% rosiglitazone, $8 \%$ glimepiride, $8 \%$ placebo, $11 \%$ sitagliptin, $15 \%$ glargine, and $25 \%$ exenatide). This is a clinically relevant endpoint, given that many antidiabetic therapies increase body weight and risk of 
hypoglycemia. The composite endpoint also aligns nicely with CER and is a reasonable way to compare antidiabetic therapies, yielding some indication of risk-benefit.

Because liraglutide caused dose-dependent and treatmentduration-dependent thyroid C-cell tumors at clinically relevant exposures in both genders of rats and mice, the liraglutide label has a boxed warning. It is not known whether liraglutide causes these tumors in humans, but liraglutide is contraindicated in patients with a personal or family history of medullary thyroid cancer and in patients with multiple endocrine neoplasia syndrome type $2 .^{4}$ No cases of medullary thyroid cancer were observed in patients treated with liraglutide in the 6 phase 3 clinical trials of 26- to 52-weeks duration.

Liraglutide also has a Risk Evaluation and Mitigation Strategies (REMS) program including an FDA safety warning on June 13, 2011, regarding the risk of thyroid cancer and pancreatitis with the recommendation to "observe patients carefully for signs and symptoms of pancreatitis (including persistent severe abdominal pain, sometimes radiating to the back, and which may or may not be accompanied by vomiting)" after use of liraglutide and after dose increases. ${ }^{16}$

Additional interpretation of CER data for antidiabetic drugs in general and the GLP-1 agonists in particular, including liraglutide, were presented in the Agency for Healthcare Research and Quality (AHRQ) comparative effectiveness review on antidiabetic agents, released in March 2011. ${ }^{17}$ This CER report included 5 studies with liraglutide as a comparator. For Alc reduction, 3 randomized controlled trials (RCTs) found "conflicting results" in comparison of sulfonylureas with liraglutide; 1 small RCT found no difference in Alc reduction, and the 2 larger RCTs favored liraglutide, but 1 of the 2 larger RCTs "underdosed the sulfonylurea [glibenclamide] arm." 17 The 3 RCTs were not combined in a meta-analysis due to dosing differences within and between studies. The small phase 2 RCT that found no difference in Alc reduction compared liraglutide (0.045 mg, $0.225 \mathrm{mg}, 0.45 \mathrm{mg}, 0.60 \mathrm{mg}$, or $0.75 \mathrm{mg}$ ) with glimepiride (1-4 mg); the changes in Alc were comparable with glimepiride at the 2 highest dosage levels of liraglutide. ${ }^{18}$ The effect on glycemic control with liraglutide was superior to glimepiride in the other 2 RCTs. ${ }^{9} 19$ The larger RCT that "underdosed" the sulfonylurea was conducted in Japan and compared glibenclamide $2.5 \mathrm{mg}$ per day with liraglutide at 0.9 mg per day. ${ }^{19}$

Although 3 studies showed weight loss with liraglutide and weight gain with sulfonylurea, both relative to baseline body weight, the AHRQ CER found a 2-fold higher rate of adverse gastrointestinal events in liraglutide patients ( $50 \%$ vs. 26\% for the sulfonylurea glimepiride) and rates of nausea, vomiting, and diarrhea approximately 2-3 times higher for liraglutide versus glimepiride, consistent with the known adverse effects profile of GLP-1 receptor agonists.

\section{Need to Confirm RCT Findings in the Real World}

According to roundtable participants, when making a decision regarding whether and how to add a drug to the formulary, efficacy, safety, and cost are the most important considerations. If a new product comes to the market with strong differentiating data, including head-to-head trials, those data will improve access to the formulary. However, the concern remains that results in clinical trials do not translate into meaningful differences in the real world. It is critical that post-market data be made available as soon as possible after launch.

Decision makers want to be certain that what happened in the RCTs is also happening in their plans' memberships. Roundtable participants suggested that the manufacturer should therefore use opportunities to validate RCT results in real-world studies. The advantages and disadvantages of clinical trial data versus real-world data have been contrasted by a few recent publications on a general basis or, specifically, for diabetes. ${ }^{20}$

As noted by Schneeweiss et al. (2011), the role of efficacy data from clinical trials and comparative effectiveness data from observational studies changes over a drug's life cycle, with RCT and placebo trials more important in the early phases, and CER becoming more important late in phase 3 and particularly in phase 4 studies. ${ }^{21}$ As Schneeweiss et al. point out, there are a number of methodological challenges in using post-marketing observational data for CER. These include bias such as confounding at the physician and patient level, sparse data during early marketing, and disease and data issues. Disease issues include the increased likelihood that first-in-class medications will be used in patients with more severe disease, and the long lag-time between product launch and the development of the disease in patients for a drug that is designed to prevent that disease. A number of data issues also are important and include the lag time for data to be available, lack of necessary granularity of information, and lack of data on suitable outcome measures. ${ }^{21}$

A major initial challenge is selective prescribing (or channeling) when a new drug enters the market. Patients who are well controlled and tolerating existing agents are much less likely to be switched to a new agent than patients who are not. Therefore, these early patients are not likely to be representative of patients who will end up taking the drug later. ${ }^{10}$ For example, when exenatide came to market, Segal et al. (2007) showed that early users (those who began taking exenatide in its first 3 months) had higher Alc levels and were more likely to have taken insulin and other oral antidiabetic drugs than patients who began exenatide 6 to 7 months after introduction. ${ }^{22}$ Sparse data available close to launch causes several problems, including a small sample size and difficulty in controlling for bias due to small numbers. 
Schneeweiss et al. propose several solutions to these problems. ${ }^{21}$ Although most pre-marketing clinical trials use placebo controls, more are being done with active comparators as well, as was the case with liraglutide and the LEAD trial program. Simulations using simulation software may also be of value. Some health plans, such as Kaiser Permanente, use software such as Archimedes in making coverage decisions when data are limited. ${ }^{23}$

The roundtable participants came to the conclusion that observational studies using large health care databases to assess treatment effectiveness in patients encountered in day-to-day clinical practice can complement RCTs that are conducted prospectively in multiple sites with large patient numbers. Use of outcomes from observational studies, which include larger and more diverse patient populations with common comorbidities and longer follow-up periods, can expand upon RCT data. Finally, identification of clinically important differences between therapeutic options and data on long-term drug effectiveness and safety can result from well-designed observational studies. ${ }^{14}$

Although real-world data are considered to be important, initial formulary decisions for new products have to be made on the basis of the clinical trial results before additional real-world data become available. Likewise, economic data are important, but the majority of such data come from observations of realworld resource utilization and thus are not available at the time formulary decisions need to be made. For example, the incidence and importance of a drug's safety concerns, such as the boxed liraglutide label warning of thyroid cancer and the REMS program and recommendation for clinicians to monitor liraglutide initiation and dose increase for symptoms of pancreatitis, can only be judged after longer use in larger patient populations and will also be matters for real-world observation.

Approaches to better understanding of patient subpopulations are usually observed with interest by decision makers. The elderly are an important subgroup in diabetes, and data on liraglutide in this subpopulation are available. Data from a pooled analysis of 6 randomized, placebo-controlled multinational trials include data on 797 patients aged 65 years or older, or about $20 \%$ of the patient population. Between $57 \%$ and $67 \%$ of patients on liraglutide $1.2 \mathrm{mg}$ and $1.8 \mathrm{mg}$ per day reached their Alc goals with no difference in response between those less than 65 and older patients. There also was no difference in the amount of weight lost or in the nature and frequency of adverse events between the age groups. ${ }^{24}$ If an algorithm for identifying patients with a high chance of good response were developed by this approach and could be used to triage patients based on their potential responses to the existing therapies, the approach to future therapy could be changed fundamentally. Using such an algorithm could drive patients toward the optimal therapy. A higher degree of personalization created by using an algorithm that predicts improved chances for response to therapy would improve health outcomes and, therefore, the value of the therapy. If differences are found in behaviors among the patients responding or not responding to therapy, such findings could be used to target behavioral programs and support patients at risk for inferior outcomes.

\section{Opportunities for Additional Comparative Data}

Even if a drug comes to market with a solid set of CER data, some roundtable participants felt that additional comparative data might even strengthen the case for the drug. For example, additional studies might show whether a new drug might benefit subpopulations, such as patients with previously inadequately controlled diabetes, or additional studies could pursue alternative combination therapies or other comparators. For example, participants suggested that studying the use of liraglutide plus insulin presented an interesting opportunity and relevance for use of the product outside of available clinical trial data. Rosenstock et al. (2011) recently presented data in a poster abstract that described evaluation of the addition of insulin detemir to patients on metformin and liraglutide $1.8 \mathrm{mg}$ daily who had not achieved the Alc goal of $<7 \%$. An additional $0.5 \%$ decrease in Alc was achieved, and $43 \%$ of the patients not at goal reached goal. Hypoglycemia rates were low and no major hypoglycemia events occurred..$^{25}$ Such data validated across large health plan populations would be useful.

The expectations of formulary decision makers frequently differ from those of regulators regarding the endpoints used in clinical studies and observational studies. Formulary decision makers note that Alc is still only a surrogate marker for adults with type 2 diabetes, and significant Alc reduction in a clinical study does not necessarily equate to satisfactory long-term control of diabetes and related negative cardiovascular outcomes, despite strong evidence that Alc reduction is associated with microvascular risk reduction.

The concept of disease modification might open a new, very attractive set of therapeutic opportunities. Studies on disease progression, disease reversion, beta-cell function and insulin sensitivity would be of major interest for all stakeholders in the field of type 2 diabetes, including patients with pre-diabetes, providers, policymakers, and payers.

Another interesting opportunity recognized by the thought leaders addressed whether the accrual of outcomes (such as cardiovascular improvement in type 2 diabetes) could be shown to outweigh the increased drug costs. This is particularly an issue since the direct drug cost of GLP-1 receptor agonists such as liraglutide is much higher than the oral agents used to treat type 2 diabetes. Such data would definitely support price and reimbursement decisions. The LEADER (Liraglutide Effect 
and Action in Diabetes: Evaluation of cardiovascular outcome Results, NCT01179048) trial will address this with the primary composite outcomes of cardiovascular death, nonfatal myocardial infarction, and nonfatal stroke. ${ }^{26}$

With any drug, information on long-term use is desired. Whereas an RCT may provide such data, the results are often not available for 5 to 10 years after the launch of a drug. Evaluation of long-term outcomes using real-world data can often provide results much sooner. Not only do such data show long-term outcome trends, but they can be used to resolve safety issues such as the boxed warning for liraglutide and the additional label warning regarding renal impairment added 14 months after initial FDA approval. The decision makers want the option of proactively managing risks instead of having to react to them. Therefore, ongoing information concerning the safety of a drug is essential. However, it is clear that potential risks can be confirmed or countered only after many patients have been treated with the drug.

Recently Ahmann (2011) has summarized the data on new classes of antidiabetic drugs (incretin-based agents including dipeptidyl peptidase-4 [DPP-4] inhibitors and GLP-1 receptor agonists) and application of CER to drugs used to treat type 2 diabetes mellitus. Ahmann determined that ongoing CER can help guide individualized, patient-centered treatment of patients with type 2 diabetes mellitus and potentially reduce trial-and-error therapy. He also said that CER can help identify which treatments would be preferred for patient subgroups and in patients at high risk of developing specific adverse events. ${ }^{27}$ In the area of type 2 diabetes, for example, patients with renal impairment, cardiovascular disease and increased age may have been excluded from the clinical trial program, but they are prevalent patient types. Data documenting the positive impact of a therapy on health and cost outcomes in patients with multiple morbidities can support the drug's placement in the formulary and provide useful information to those patient groups and their health care providers. As Ahmann suggests, the best way to make CER useful in diabetes is to further encourage head-to-head clinical and observational studies to generate high-quality evidence that can be incorporated into CER evaluations. ${ }^{27}$

\section{Conclusions}

Liraglutide came to the market with unusually robust data demonstrating clinical comparative efficacy and safety. The thought leaders involved in the roundtable discussion agreed that well-planned trial designs that result in a broad range of relevant data, such as that presented for liraglutide, can provide a good basis for the decision maker. There are frequently challenges due to the lag time between trial design, completion and market availability. New competitors into the marketplace and changes in treatment guidelines sometimes make it difficult to obtain relevant CER data.

Based on the roundtable discussion, the thought leaders had several recommendations for any manufacturer implementing CER. First, manufacturers should translate clinical and CER data into tools that will enable decision makers to create effective benefit designs and make good formulary decisions. Second, manufacturers should be proactive in communication with all stakeholders, including decision makers, keeping them informed of further developments, information, or new evidence gathered, especially with safety issues. Manufacturers should pursue opportunities to validate data from the RCT program in the real world. Manufacturers should pursue methods to better identify patients who will best respond to their products. And finally, the roundtable participants recommend that manufacturers perform CER along the lines envisioned by the federal government.

\section{Authors}

GARY ODERDA, PharmD, MPH, is Professor and Director, Pharmacotherapy Outcomes Research Center, University of Utah, College of Pharmacy, Salt Lake City, Utah. S. MONET SIFFORDWILSON, MD, is Medical Director, Victoza, Novo Nordisk Inc., Princeton, New Jersey.

AUTHOR CORRESPONDENCE: Gary Oderda, PharmD, MPH, Pharmacotherapy Outcomes Research Center, University of Utah, College of Pharmacy, 421 Wakara Way, Ste. 208; Salt Lake City, UT 84108. Tel.: 801.581.6257; E-mail: Gary.Oderda@pharm.utah.edu. S. Monet Sifford-Wilson, MD; Novo Nordisk Inc., 100 College Rd. W., Princeton, NJ 08540. Tel.: 609.786.4446; E-mail: shsw@novonordisk.com.

\section{DISCLOSURES}

This JMCP supplement was sponsored by Novo Nordisk Inc. and prepared by the Millcreek Outcomes Group (Salt Lake City, Utah). Gary Oderda has served as a consultant for Pacira and Novo Nordisk and as a speaker for Jansen; he has done funded research for King (Pfizer), Novartis, and Takeda. S. Monet Sifford-Wilson is a full-time employee of Novo Nordisk Inc. and shareholder of Novo Nordisk Inc.

\section{ACKNOWLEDGEMENTS}

The authors thank Anke-Peggy Holtorf, PhD, MBA, for her assistance in gathering and preparing the data used in this article, and Kelley $\mathrm{J}$. P. Lindberg, BS, for writing assistance in preparation of this manuscript. 


\section{ROUNDTABLE PARTICIPANTS}

Diana I. Brixner, PhD, RPh, and Gary Oderda, PharmD, MPH, served as the moderators for the live roundtable meeting. All 9 participants in the roundtable process agreed to be identified: John J. Barron, PharmD (HealthCore, Inc., Wilmington, DE); Cathryn A. Carroll, PhD, MA, MBA, BSPharm (Two Rivers Behavior Health System, Kansas City, MO); Scott L. Charland, PharmD, FCCP (Medco Research Institute, LLC, a wholly owned subsidiary of Medco Health Solutions, Inc., and Adjunct Associate Professor, Department of Clinical Pharmacy, School of Pharmacy, University of Colorado, Winter Park, CO); Rhonda A. Driver, BSc Pharm, RPh (MO HealthNet Division, Missouri Department of Social Services, Jefferson City, MO); Jeffrey D. Dunn, PharmD, MBA (SelectHealth, Salt Lake City, UT); William H. Francis, MBA, RPh (The University of Arizona Health Plans, Tucson, AZ); Raulo S. Frear, PharmD (RegenceRx, Boise, ID); James A. Jorgenson, RPh, MS, FASHP (Indiana University Health, Indianapolis, IN); and Hau Le, RPh, MSc (PharMerica [in October of 2010], Salt Lake City, UT).

In addition, the following individuals participated as reactor panelists: Ingrid Ma, PharmD, RPh (Galena, OH); John B.Watkins, PharmD, MPH, BCPS (Premera Blue Cross, Mountlake Terrace, WA); and T. Jeffrey White, PharmD, MS (Costa Mesa, CA).

\section{REFERENCES}

1. Saydah SH, Fradkin J, Cowle CC. Poor control of risk factors for vascular disease among adults with previously diagnosed diabetes. JAMA. 2004;291(3):335-42. Available at: http://jama.ama-assn.org/content/291/3/335.full.pdf+html. Accessed April 30, 2012.

2. Ong KL, Cheung BM, Wong LY, Wat NM, Lam KS. Prevalence, treatment and control of diagnosed diabetes in the U.S. National Health and Nutrition Exam Survey 1999-2004. Ann Epidemiol. 2008;18(3):222-29.

3. Hochman M, McCormick D. Characteristics of published comparative effectiveness studies of medications. JAMA. 2010;303(10):951-58. Available at: http://jama.ama-assn.org/content/303/10/951.full?home. Accessed April 30, 2012

4. Victoza (liraglutide) injection. Novo Nordisk. April 2012. Available at: http://www.novo-pi.com/victoza.pdf. Accessed April 30, 2012.

5. Clement FM, Harris A, Li JJ, Yong K, Lee KM, Manns BJ. Using effectiveness and cost-effectiveness to make drug coverage decisions: a comparison of Britain, Australia, and Canada. JAMA. 2009;302(13):1437-43. Available at: http://jama.ama-assn.org/content/302/13/1437.full. Accessed April 30, 2012.

6. Chokshi DA, Avorn J, Kesselheim AS. Designing comparative effectiveness research on prescription drugs: lessons from the clinical trial literature. Health Aff (Millwood). 2010;29(10):1842-48

7. Marre M, Shaw J, Brändle M, et al.; for the LEAD-1 SU study group. Liraglutide, a once-daily human GLP-1 analogue, added to a sulphonylurea over 26 weeks produces greater improvements in glycaemic and weight control compared with adding rosiglitazone or placebo in subjects with type 2 diabetes (LEAD-1 SU). Diabet Med. 2009;26(3):268-78. Available at: http:// www.ncbi.nlm.nih.gov/pmc/articles/PMC2871176. Accessed April 30, 2012.
8. Nauck M, Frid A, Hermansen K, et al.; for the LEAD-2 study group. Efficacy and safety comparison of liraglutide, glimepiride, and placebo, all in combination with metformin, in type 2 diabetes: the LEAD (liraglutide effect and action in diabetes)-2 study. Diabetes Care. 2009;32(1):84-90. Available at: http://www.ncbi.nlm.nih.gov/pmc/articles/PMC2606836/?tool=pubmed. Accessed April 30, 2012.

9. Garber A, Henry R, Ratner R, et al.; for the LEAD-3 (Mono) study group. Liraglutide versus glimepiride monotherapy for type 2 diabetes (LEAD-3 Mono): a randomised, 52-week, phase III, double-blind, parallel-treatment trial. Lancet. 2009;373(9662):473-81.

10. Zinman B, Gerich J, Buse JB, et al.; for the LEAD-4 study group. Efficacy and safety of the human glucagon-like peptide-1 analog liraglutide in combination with metformin and thiazolidinedione in patients with type 2 diabetes (LEAD-4 Met+TZD). Diabetes Care. 2009;32(7):1224-30. Available at: http://care.diabetesjournals.org/content/32/7/1224.full.pdf + html. Accessed April 30, 2012.

11. Russell-Jones D, Vaag A, Schmitz O, et al.; for the LEAD-5 Met + SU study group. Liraglutide vs insulin glargine and placebo in combination with metformin and sulfonylurea therapy in type 2 diabetes mellitus (LEAD-5 met+SU): a randomised controlled trial. Diabetologia. 2009;52(10):2046-55. Available at: http://www.ncbi.nlm.nih.gov/pmc/ articles/PMC2744824/?tool=pubmed. Accessed April 30, 2012.

12. Buse JB, Rosenstock J, Sesti G, et al.; for the LEAD-6 study group. Liraglutide once a day versus exenatide twice a day for type 2 diabetes: a 26-week randomised, parallel-group, multinational, open-label trial (LEAD6). Lancet. 2009;374(9683):39-47.

13. Pratley RE, Nauck M, Bailey T, et al.; for the 1860-LIRA-DPP-4 study group. Liraglutide versus sitagliptin for patients with type 2 diabetes who did not have adequate glycaemic control with metformin: a 26-week, randomised, parallel-group, open-label trial. Lancet. 2010;375(9724):1447-56.

14. Novo Nordisk A/S. Victoza backgrounders-facts about the LEAD clinical study programme. Available at: http://www.novonordisk.com/press/ liraglutide-press-room/liraglutide-Backgrounders_Facts-about-LEADTMclinical-study-programme.asp. Accessed April 30, 2012.

15. Zinman B, Schmidt WE, Moses A, Lund N, Gough S. Achieving a clinically relevant composite outcome of an Alc of $<7 \%$ without weight gain or hypoglycaemia in type 2 diabetes: a meta-analysis of the liraglutide clinical trial programme. Diabetes Obes Metab. 2012;14(1):77-82. Available at: http://onlinelibrary.wiley.com/doi/10.1111/j.1463-1326.2011.01493.x/full. Accessed April 30, 2012.

16. U.S. Food and Drug Administration. Victoza (liraglutide [rDNA origin]) Injection: REMS-risk of thyroid C-cell tumors, acute pancreatitis. June 13, 2011. Available at: http://www.fda.gov/Safety/MedWatch/SafetyInformation/ SafetyAlertsforHumanMedicalProducts/ucm258826.htm. Accessed April 30, 2012.

17. Bennett WL, Wilson LM, Bolen S, et al. Oral diabetes medications for adults with type 2 diabetes: an update. Comparative effectiveness review no. 27. (Prepared by Johns Hopkins University Evidence-based Practice Center for the Agency for Healthcare Research and Quality under contract no. 290-02-0018 .) AHRQ Publication No. 11-EHC038-EF. Rockville, MD: Agency for Healthcare and Research Quality. March 2011. Available at: http://www.effectivehealthcare.ahrq.gov/ehc/products/155/644/CER27_ OralDiabetesMeds_20110623.pdf. Accessed April 30, 2012. 
18. Madsbad S, Schmitz O, Ranstam J, Jakobsen G, Mathews DR; NN22111320 International Study Group. Improved glycemic control with no weight increase in patients with type 2 diabetes after once-daily treatment with the long-acting glucagon-like peptide 1 analog liraglutide (NN2211): a 12-week, double-blind, randomized, controlled trial. Diabetes Care. 2004;27(6):1335-42.

19. Seino Y, Rasmussen MF, Nishida T, et al. Efficacy and safety of the oncedaily human GLP-1 analogue, liraglutide, vs glibenclamide monotherapy in Japanese patients with type 2 diabetes. Curr Med Res Opin. 2010;26(5):1013-22.

20. Silverman SL. From randomized controlled trials to observational studies. Am J Med. 2009;122(2):114-20.

21. Schneeweiss S, Gagne JJ, Glynn RJ, Ruhl M, Rassen JA. Assessing the comparative effectiveness of newly marketed medications: methodological challenges and implications for drug development. Clin Pharmacol Ther. 2011;90(6):777-90.

22. Segal JB, Dy SM, Millman EA, Herbert R, Bass EB, Wu A. Diffusion into use of exenatide for glucose control in diabetes mellitus: a retrospective cohort study of a new therapy. Clin Ther. 2007;29(8):1784-94.
23. Dudl RJ, Want MC, Wong M, Bellows J. Preventing myocardial infarction and stoke with a simplified bundle of cardioprotective medications. Am J Manag Care. 2009;15(10):e88-e94. Available at: http://www.dmhc.ca.gov/ library/reports/news/allphase.pdf. Accessed April 30, 2012.

24. Bode BW, Brett J, Falahati A, Pratley RE. Comparison of the efficacy and tolerability profile of Liraglutide, a once-daily human GLP-1 analog in patients with type 2 diabetes $>65$ and $<65$ years of age: a pooled analysis from phase III studies. Am J Geriatr Pharmacother. 2011;9(6):423-33.

25. Rosenstock J, Devries JH, Seufert J, et al. A new type 2 diabetes treatment paradigm: sequential addition of liraglutide to metformin and then basal insulin detemir [abstract]. Diabetes. 2011;60(Suppl 1):A76.

26. Clinicaltrials.gov. Liraglutide effect and action in diabetes: evaluation of cardiovascular outcome results - a long-term evaluation (LEADER). NCT01179048. Last updated April 17, 2012. Available at: http://clinicaltrials. gov/ct2/show/NCT01179048. Accessed April 30, 2012.

27. Ahmann A. Application of comparative effectiveness research in evaluation of treatments for type 2 diabetes mellitus. Am J Manag Care. 2011;17(2 Suppl):S41-S51. 